\title{
Smart e-Commerce Recommendation System Using Data Mining Techniques
}

\author{
Adithya Gupta, Ankitha A, Subramanya Raju, Vidya B H, Rumana Anjum* \\ Department of Computer Science and Engineering, Vidyavikas Institute of Engineering and \\ technology, Mysuru, India
}

DOI: https://doi.org/10.21467/proceedings.1.68

* Corresponding author email: rumanaanjum85@gmail.com

\begin{abstract}
In existing e-commerce recommendation system it's harder for the consumer to find the product they want. Existing recommendation system uses traditional algorithms which Focus only on user ratings data, which affect the quality of system's recommendation. The improvement consider in this paper mainly refer to limited resource, data valid time, cold start problem and frequent item set which are not well considered in existing ecommerce recommendation system. To solve the above mentioned problem a algorithm based on limited resource and a solution to cold start problem are proposed.
\end{abstract}

\section{INTRODUCTION}

With the development of existing e-commerce system, the structure of structure of ecommerce system became more and more complex. To develop a recommender system, the collaborative filtering is the best known approach which considers only the ratings of users who have similar ratings patterns. The major challenge of the collaborative filtering approach can be how to Make recommendations for a new user, that is called cold start problem. Ecommerce recommendation is to take advantage of e-commerce site to provide information and suggestions, to help consumers make purchasing decisions. Although the e-commerce recommendation system has been successful, it faces challenges with the development of ecommerce. Compare to existing e-commerce recommendation system, following factors should be taken into account.

(C) 2018 Copyright held by the author(s). Published by AIJR Publisher in Proceedings of the $3^{\text {rd }}$ National Conference on Image Processing, Computing, Communication, Networking and Data Analytics (NCICCNDA 2018), April 28, 2018.

This is an open access article under Creative Commons Attribution-NonCommercial 4.0 International (CC BY-NC 4.0) license, which permits any non-commercial use, distribution, adaptation, and reproduction in any medium, as long as the

AijR license, which permits any non-commercial use, distribution, 
1) Limited resource: Personal recommendation for limited product with special lowest price in special offer period. Those kinds of should be recommended with priority based on their transaction history, so that customers can buy with pleasure.

2) Datavalid time: Here, the records which were recorded long time ago will not be taken for recommendation of products. Because those records are not accurate anymore. Only the records which were recorded recently will be allowed to use for recommendation.

3) Cold start problem: If the user is new to the website then it will be a problem for him to find the product which he wants as he has no transaction history.

4) Frequent item set: Which uses apriori algorithm for designing operation databases containing transactions (for example collection of items bought by a customer or details of website frequentation).

\section{LIMITED RESOURCE}

The trading volume can be enhanced if the products with special price recommended to specific customers properly. High -frequency special sale events enhance the attention of customers and trading volume. Special-offer products have characteristics of lower price and limited amount. So, it's better to record those special-offer products in a table in database in advance. Usually the record list of special-offer products should be recorded automatically by recommendation system when seller modify the price.

In addition, the purchasing probability of limited special-offer product i $(X i)$ is a critical data for recommendation system. Those products with extremely low probability, which is meaningless, are not expected to be recommended. The purchasing probability of product i(Xi) can be defined as follow: $X i=Y i / Z i$.

Where, $Y i$ is the number of attention of product $\mathrm{I}, \mathrm{Zi \text {is }}$ the stock of the product $\mathrm{i}$.

\section{COLD START}

It is a problem for a customer who visits the website for the first time to get the product they want. There are some basic solutions to solve this problem. So, some basic information of user should be taken into account, such as location, registration

time. Users from same place, with similar purchasing needs. If recommendation can be given out recommendation system, according to similar user's purchase, it would be helpful for new user. According to description above, a solution can be proposed which mainly contains 4 steps:

- Obtain the location of user based on GPS and get the registration time of target user.

- Statistics users in same location who registered within 1 year and their purchasing history.

- Calculate the trading volume of each product in the statistical results.

Proceedings of the $3^{\text {rd }}$ National Conference on Image Processing, Computing, Communication, Networking and Data Analytics (NCICCNDA 2018) 
- Sorted by trading volume and get top 10 products as recommendation products.

\section{HYBRID APPROACH BASED ON LIMITED RESOURCE}

Here, we use hybrid algorithm to recommend the products to a particular user when the products are limited. There are 2 steps to follow.

1. Scan the user interaction records and determine the support for all the items.

Support $[\mathrm{p}]=$ No. of transaction containing product $\mathrm{p}$

No. of total transaction

2. Generation of neighbors

Suppose that the set of all consumers $S=\{S 1, S 2 \ldots . . . S n\}$, for each consumer $\mathrm{Si}(\mathrm{i}=1,2 \ldots \ldots \mathrm{n})$, the system can calculate the neighbors list including the top $\mathrm{N}$ consumers which similarity is higher than the given threshold.

\section{FREQUENT ITEM SET}

It uses an apriori algorithm for designing operation databases containing transactions(For example, collection of items bought by a customer or details of website frequentation). Support: The percentage of task relevant data transaction for which the pattern is true. Support (product1 $\rightarrow$ product2) can be calculated as follows.

No. of transactions containing product 1 and product 2

No. of total transactions

Confidence: The measure of certainty or trustworthiness associated with each discovered pattern. Confidence(product1 and product2) can be calculated as follows.

No. of transaction containing both product 1 and product 2

No. of transaction containing product 1

By using above 2 formulae we can calculate support and confidence for the product recommendation. Where support is the percentage of task relevant data transaction for which the pattern is true. And confidence is defined as the measure of certainty or trustworthiness associated with each discovered pattern where transaction containing both product1 and product 2 where as to calculate support total no of transaction will be considered.

\section{CONCLUSION}

With the development of e-commerce, personalized recommendation has been paid more and more attention. Limited resource situation, data valid time, cold start problem and frequent item set have not been well considered in existing e-commerce recommendation system. This paper proposes limited resource table method, an algorithm based on limited resource and a solution to cold start problem, which can enhance the effect of recommendation system. The solutions proposed in this paper are meaningful for e-commerce websites and recommendation system. In the future, more and more factors will be taken into account in e-commerce recommendation system. 


\section{REFERENCES}

[1] Deng Ailin, Zhu Yangyong, Shi Bole. A collaborative Filtering Recommendation algorithm based on item rating prediction[j]. Journal of software. 2003(09)

[2] Resnick p, Varian HR. Recommender systems. Communications of the ACM. 1997

[3] Jiang shaowei. Recommendation models Based on competitive Relationship[D].

[4] Wang Guoxia, Liu Heping. Survey of personalized recommendation system[J]. Computer Engineering and Application, 2012, 48(47):66-76. DOI:10.3778/J.ISSN.10002-8331.2012.07.018.

[5] G. Adomavicius, A. Tuzhilin. Toward the next generation of recommender system[J]. IEEE Transactions on knowledge and data Engineering, 2005, 17(06): 734-749, DOI:10.1002/PON.1586

[6] Sum Dongting, He Tao, Zhang Fuhai. Survey of Cold Start Problem In Collaborative Filtering Recommender System[J]. Computer and Moderinization,2012,(5)

Proceedings of the $3^{\text {rd }}$ National Conference on Image Processing, Computing, Communication, Networking and Data Analytics (NCICCNDA 2018) 